\title{
Visibilidad internacional de la producción científica iberoamericana en biblioteconomía y documentación (1991-2000)*
}

\author{
Félix de M oya-A negón \\ felix@ugr.es \\ V íctor H errero-Solana \\ victorhs@ugr.es \\ www.ugr.es/ victorhs
}

\section{Resumen \\ Se analiza la producción iberoamericana en biblioteconomía y documentación existente en el Social Science Citation Index para el periodo 1991-2000. Se analiza la producción por países, instituciones, revistas y autores. También se estudia la citación de autores y revistas. Por último, se realiza un análisis de cocitación de revistas (ACR) y se representa mediante un mapa bidimensional. \\ Palabras-clave}

Análisis de dominio; Bibliometría; Iberoamérica; Biblioteconomía y documentación.

International visibility for the scientific production of lberoamerica in Library and Information Science (1991 - 2000)

\begin{abstract}
We study the production of iberoamerican Library and Information Science in the Social Science Citation Index from 1991 to 2000. We analyze the production by contries, institutions, journals and authors. We also work with authors and journals citation. Finally we make and journal cocitation analysis (JCA) and show by a bidimensional map.
\end{abstract}

Keywords

Domain analysis; Bibliometrics; Iberoamerica; Library and information science.

\section{INTRODUCCIÓN}

El campo temático correspondientealaBiblioteconomía y $D$ ocumentación ( $B \& D$ ) es quizás el más estudiado a través técnicas bibliométricas, particularmentemediante la cocitación de autores (ACA) y de revistas (ACR). A nivel internacional hay gran cantidad detrabajos, sobre los cual es destaca, a nuestro juicio, el anál isis de dominio White y M cC ain ${ }^{1}$. También se realiza un análisis de dominio basado en técnicas conexionistas en otros dos trabajos ${ }^{2,3}$. Por último, debemos destacar el trabajo anterior de Persson sobre la producción de la revista J ournal of theAmerican Society for I nformation Science(J asis), uno de los trabajos pioneros en estesentido ${ }^{4}$.

En nuestra región también se han realizado análisis dela literatura sobre $B \& D$, aunque en la mayoría de los casos se han utilizado fuentes de información de la propia región. C on respecto a la investigación española son de destacar los trabajos de Moya A negón y Jiménez C ontreras, queanalizan los patrones de autoría ${ }^{5}$ y realizan un $\mathrm{A} \mathrm{CA}^{6}$ dela producción deeste país. C on relación ala producción latinoamericana, la literatura es al go escasa, y solo encontramos al gunos trabajos como por ejemplo un análisis de la producción de la revista mexicana Investigacion Bibliotecologica?

El presente trabajo tiene como objetivo brindar una visión global delaproducción científicaiberoamericana (Latinoamérica + España y Portugal), visible internacionalmente, en $B \& D$. Entendemos aquí por visibilidad internacional de la producción científicala que se logra a través de trabajos en los que participan autores iberoamericanos y que se publican en revistas que son analizadas en bases de datos internacionales, en este caso el Social Science C itation I ndex (SSCI). Es importante destacar que la mayoría de las revistas especializadas en $B \& D$ delaregión, no son analizadas en

\footnotetext{
* U na versión preliminar de este trabajo, correspondiente al periodo 1991-98, fue presentada en el V Encuentro de E D I B C I C , G ranada, 21 al 25 de febrero de 2000.
} 
esta base de datos, cuyo sesgo se inclina hacia el mundo anglosajón. D ehecho, en el presenteestudio solamente una de las revistas anal izadas es iberoamericana, aunque no pertenece estrictamente a $B \& D$, pues tiene la consideración de multidisciplinar.

Esto es importantede destacar ya que de estaformano se estará estudiando la investigación iberoamericana en $B \& D$, sino la porción de esta última que ha sido homologadainternacionalmente. C omo veremos más adelante parece haber algunas áreas temáticas que predominan sobre otras y quetienen mayor visibilidad a nivel mundial. U no delos objetivos del presenteestudio consiste en determinar cuales son estas áreas.

\section{MÉT O D O SY MATERIALES}

Como fuente de datos para el estudio se ha utilizado la versión en C D RO M de la base Social Science C itation Index (SSC I-CD E), correspondiente al periodo 199199, aunque este último no se encuentra completo. Dentro del SSCI existe una categoría temática denominada "I nformation Science\& Library Science". Lamentablemente, la información sobre categoría temática (SC) solo está completa en la versión en línea de la base de datos y no en el CDRO M. N o obstante, pudimos reconstruir la categoría "I nformation Science \& Library Science" a partir de los títulos de las revistas que aparecen en el J ournal C itation Reports (JCR ). En un principio nos basamos en el JCR de 1998, pero también incluimos los títulos de las ediciones de 1996, 1994 y 1992. Lalista completa de revistas puede apreciarseen el apéndice A . D elalistaoriginal eliminamos la revista Social Science I nformation Sur les Sciences Sociales, ya que la temática tratada en ella no estaba relacionada con la $B \& D$. También se eliminaron al gunos artículos deTelecommunicationsPolicy. En contrapartida se decidió agregar selectivamente la producción de Interciencia, una revista de la región que pese a figurar bajo la categoría temática "Multidisciplinar", contiene varios artículos sobre bibliometría. La elección de esta se debió a las citas obtenidas desde revistas de la categoría como Scientometrics.

Laselección delaproducción por países de la región no tuvo inconvenientes, salvo en el caso de Puerto Rico, que no está considerado como un país sino que aparece como un estado de Estados U nidos. Esto se soluciona utilizando la ecuación "U SA AN D PR", yaquePR es el código de estado de Puerto Rico. N o obstante, fue necesario depurar el resultado yaqueesta solución no es exacta debido a que entran algunos registros que no corresponden aPuerto Rico.

L os registros obtenidosfueron "bajados" con formato de etiquetas de dos caracteres, para ser procesados mediante el software Bibexcel desarrollado por O lle Persson. Bibexcel es un programa desarrollado específicamente para la manipulación y transformación de registros bibliográficos. El resultado de dichas transformaciones puede ser procesado a su vez mediante el M S-Excel, 0 cualquier otro programaquetrabajecon formato detexto plano en columnas. Bibexcel permite combinar la información extractada de diferentes campos de un registro, real iza conteos por frecuencia, co-ocurrencias dediversos el ementos, y emparejamiento bibliográfico (bibliographic coupling). A demás deestastareas, cuentacon un procedimiento para encontrar enlaces ( links) decitas entre diferentes documentos de un conjunto determinado. Bibexcel es un programa de dominio público que puede obtenerse en Internet (http:// www.umu.se/inforsk/).

A ntes de realizar los conteos definitivos fue necesario realizar un fuerte control de autoridades en diferentes campos: autor (A U ), institución (C S), referencia citada (CR). El control más complejo fueel delas instituciones, paralo cual nos valimos del "D irectorio deU niversidades I beroamericanas" realizado por el Vicerrectorado de Investigación y Relaciones Internacionales de la U niversidad de G ranada (http:// www.ugr.es/ ri/). C on los otros campos no hubo tanto problema, salvo en el caso de los nombres de las revistas de la región, los cuales debieron ser minuciosamente comprobados.

Lainformación obtenidadel SSCI secomplementarácon la de una base de datos de la especial idad: I nformation Science A bstracts (ISA). Si bien esta base de datos no recoge las citas de los trabajos, incluye la filiación institucional del primer autor. Esto permite apreciar el volumen de producción de la región en el concierto mundial.

\section{AN ÁLISIS DE RESULTADOS}

A continuación se comentan los resultados obtenidosa partir de la fuente de datos, divididos en epígrafes temáticos. En algunos casos se consignarán las dificultades encontradas a la horade obtenerlos, así como también los criterios empleados para su selección. 


\section{PRODUCCIÓN POR PAÍS}

En el cuadro 1 vemos el detalle de los val ores correspondientes a cada país en ambas bases de datos, ISA y SSC I, estando esta última dividida en la cantidad total a lo largo de toda la basey específicamente lo queconcierne $a B \& D$. En primer lugar se aprecia una alta correlación entre $B \& D$ y latotalidad del SSCI, con laexcepción quizás deA rgentina, cuyaproducción en $B \& D$ no estáalaaltura desu posición en toda la base. En la figura 1 (apéndice) podemos ver laconfrontación de los val ores porcentuales de cada país de la región. En líneas generales hay una buena correlación entre los dos valores, salvo para el caso de $A$ rgentina, cuya producción en $B \& D$ no parece estar a la al tura dela producción total. Españaparecetener unaposición en $B \& D$ mucho mejor quesu mediaen $\mathrm{SSCI}$, todo lo contrario queBrasil y M éxico, quesehallan por debajo.

En la figura 2 (apéndice) vemos el gráfico de sectores correspondientes a la producción por países en $B \& D$ $\mathrm{SSCI}$, con un fuerte predominio deEspaña sobreel resto de la región, que le permite acumular casi la mitad de la producción total. Brasil y M éxico, por su parte, suman un cuarto del total quedando el cuarto restante parael resto de países.

Si utilizamos la base de datos ISA, la relación entre los países es un tanto más equilibrada. Como podemos apreciar en la figura 3 (apéndice) España y Brasil se encuentran bastante igualados, por encima del $30 \%$. EI resto de los países parece que se acomodan de forma similar al SSCI. La explicación de este fenómeno se encuentre quizás en el hecho de que los valores deISA son más representativos del volumen productivo de Brasil, sin embargo, la producción española pareceser de más calidad ya que se coloca con mayor facilidad en revistas ISI.

\section{IBER O AMÉRICA EN EL CONTEXTO IN TERNACIONAL}

En el cuadro 2 hemos añadido la información sobre producción total en $B \& D$ de otros países, con el fin de realizar un análisis comparativo. I ndudablemente, los países centrales tienen una producción superior ala de la región, colocándose España apenas hacia el final de este grupo. Lo cierto es que los países anglosajones se encuentran sobredimensionados en el SSCI, ya que indudablemente el volumen de producción está directamente relacionado con la cantidad de revistas indizadas de cada país $s^{8}$ L o que puede resultar útil es la comparación de los países dela región con otros deotras regiones periféricas, teniendo en cuentalas ventajas que tienen en este sentido los países de habla inglesa.
CUADRO 1

\section{Producción por países ISA y SSC I}

\begin{tabular}{|l|r|r|r|r|r|r|}
\multicolumn{1}{|c|}{ País } & \multicolumn{2}{|c}{ ISA } & \multicolumn{2}{c|}{ B\&D } & \multicolumn{2}{c|}{ SSCI } \\
\hline Espetía & 403 & $34,3 \%$ & 136 & $47,5 \%$ & 6450 & $42,1 \%$ \\
\hline Brasil & 394 & $33,5 \%$ & 41 & $14,3 \%$ & 2566 & $18,6 \%$ \\
\hline México & 133 & $11,3 \%$ & 33 & $11,5 \%$ & 2384 & $15,5 \%$ \\
\hline Chile & 47 & $4,0 \%$ & 15 & $5,2 \%$ & 622 & $4,0 \%$ \\
\hline Portugal & 77 & $6,5 \%$ & 14 & $4,0 \%$ & 687 & $4,5 \%$ \\
\hline Venezuela & 23 & $1,9 \%$ & 12 & $4,2 \%$ & 355 & $2,3 \%$ \\
\hline Cuba & 34 & $2,9 \%$ & 8 & $2,5 \%$ & 167 & $1,1 \%$ \\
\hline Pucrto Rico & & & 6 & $2,1 \%$ & 369 & $2,4 \%$ \\
\hline Argentina & 28 & $2,4 \%$ & 5 & $1,7 \%$ & 897 & $5,8 \%$ \\
\hline Colombia & 15 & $1,6 \%$ & 4 & $1,4 \%$ & 306 & $2,0 \%$ \\
\hline Uruguay & 5 & $0,4 \%$ & 4 & $1,4 \%$ & 57 & $0,4 \%$ \\
\hline Perú & 9 & $0,7 \%$ & 1 & $0,3 \%$ & 137 & $0,9 \%$ \\
\hline Costa Rica & 9 & $0,7 \%$ & 1 & $0,3 \%$ & 62 & $0,4 \%$ \\
\hline Ecuador & 1 & $0,1 \%$ & 1 & $0,3 \%$ & 63 & $0,4 \%$ \\
\hline
\end{tabular}

\section{CUADRO 2}

\section{Producción en $B \& D$ en diversos países del mundo}

\begin{tabular}{l||r|r|r|r|r|}
\hline Total & & 74255 & Corea del Sur & KR & 57 \\
\hline USA & US & 43120 & Irlanda & IE & 44 \\
\hline Reino Unido & UK & 2832 & Brasil & BR & 41 \\
\hline Canada & CA & 1399 & Mérica & MX & 33 \\
\hline Alemania & DE & 885 & Grecia & GR & 33 \\
\hline Rusia & RU & 411 & Bulgaria & BG & 33 \\
\hline Holanda & NL & 350 & Sudáfrica & ZA & 31 \\
\hline Australia & AU & 339 & Polonia & PL & 26 \\
\hline Francia & FR & 289 & Luxemburgo & LU & 21 \\
\hline India & IN & 181 & Portugal & PT & 18 \\
\hline Belgica & BE & 167 & Etiopia & ET & 18 \\
\hline Italia & IT & 148 & Chile & CL & 15 \\
\hline lapón & JP & 137 & Egipio & EG & 13 \\
\hline Esparia & ES & 136 & Venezuela & VE & 12 \\
\hline Israel & IL & 111 & Cuba & CU & 8 \\
\hline Dinamarca & DK & 110 & Puerto Rico & PR & 6 \\
\hline Nueva Zelanda & NZ & 105 & Argentina & AR & 5 \\
\hline Finlandia & FI & 92 & Unuguay & UR & 4 \\
\hline Hungría & HU & 89 & Colombia & CO & 4 \\
\hline Hong Kong & HK & 82 & Perí & PE & 1 \\
\hline Suecia & SE & 60 & Ectuador & EC & 1 \\
\hline
\end{tabular}

Con la base de datos ISA hemos realizado una representación por grandes regiones. En la figura 4 (apéndice) podemos apreciar el predominio deEstados U nidos y C anadá, que es equiparable a lo que encontramos en las bases del ISI. En este contexto, la producción latinoamericana es de apenas el 1\%, la mitad del porcentajequeseleadjudicaalaregión en el conjunto total de la ciencia?. 


\section{PRO DUCCIÓN POR INST IT U CIÓN}

En lafigura5 (apéndice) vemos un gráfico de barras que representala producción científica por institución. A quí se ha optado por seleccionar la institución principal, o sea la institución que aparece hasta la primera coma en el campo C S- (address). La información posterior a la primera coma (departamentos, facultades, etc.) no ha sido tendida en cuenta en este caso, pero si se incluirá más adelante cuando veamos la producción por autor.

Como se puede observar, las primeras instituciones presentan una gran producción. Si tomamos las cinco primeras llegamos a acumular el la mitad del total, tal como seveen detalleen el cuadro 3. D eestas cuatro, tres son españolas. A partir de la quinta se forma un gran grupo de instituciones con un nivel menor de producción, quevareduciéndose lentamente.

\section{PRODUCCIÓN POR REVISTA}

En la figura 6 (apéndice) y cuadro 4 vemos los valores de producción por revistafuente. Como se puedeapreciar, existe un título que comprende más de un tercio de los trabajos: la revistaScientometrics. Luego, y muy por detrás, vienen otros títulos entrelos quese encuentral nterciencia, quecomo yahemos dicho es el único propio delaregión. A I final de la lista encontramos unos 28 diferentes revistas que acumulan apenas el $22 \%$ del total. C omo veremos más adelante, existeunaciertacorrelación entre el volumen de producción en una determinadarevista con respecto a su nivel decitación.

\section{PRODUCCIÓN POR AÑOS}

En lafigura 7 (apéndice) tenemosunagráfica con la evolución histórica del periodo estudiado. Si bien la curva presenta al gunos altibajos, podemos situar la media en unos 27 trabajos por año. Si trazamos una curva de tendenciaencontraríamosquelamismatiene una tendencialigeramente positiva, aunque el valor ajuste $R^{2}$ es necesariamente bajo.

\section{PRODUCCIÓN PORAUTOR}

$C$ uando realizamos un recuento por autor, nos encontramos con una gran fragmentación de los valores obtenidos en CUADRO 4

\section{Revistas más productivas}

\begin{tabular}{|l|c|c|c|}
\hline \multicolumn{1}{|c|}{ Revista } & $\#$ & $\%$ \\
\hline Scientometrics & 99 & $33,56 \%$ \\
\hline Interciencia & 18 & $6,10 \%$ \\
\hline Journal of Information Science & 16 & $5,42 \%$ \\
\hline Journal of the American Society for Information Science & 14 & $4,75 \%$ \\
\hline IFLA Journal & 10 & $3,39 \%$ \\
\hline International Forum on Information and Documentation & 10 & $3,39 \%$ \\
\hline Journal of the American Medical Informatics Association & 8 & $2,71 \%$ \\
\hline Library Journal & 8 & $2,71 \%$ \\
\hline Libri & 8 & $2,71 \%$ \\
\hline Journal of Documentation & 7 & $2,37 \%$ \\
\hline Restaurator & 7 & $2,37 \%$ \\
\hline Knowledge Organization & 6 & $2,03 \%$ \\
\hline Information Technology and Libraries & 6 & $2,03 \%$ \\
\hline Electronic Library & 6 & $2,03 \%$ \\
\hline Otros 32 Titulos & 72 & $24,41 \%$ \\
\hline
\end{tabular}

CUADRO 3

Instituciones másproductivas

\begin{tabular}{|l|c|c|}
\multicolumn{1}{|c|}{ Institución } & $\#$ & $\%$ \\
\hline CSIC & 41 & $17,60 \%$ \\
\hline Univ Nacl Autonoma Mexico & 28 & $12,02 \%$ \\
\hline Univ Granada & 21 & $9,01 \%$ \\
\hline Univ Carlos III Madrid & 18 & $7,73 \%$ \\
\hline Univ Extremadura & 12 & $5,15 \%$ \\
\hline Univ Alcalá de Henares & 11 & $4,72 \%$ \\
\hline Univ Fed Rio de Janeiro & 9 & $3,86 \%$ \\
\hline Pontificia Univ Catolica Chile & 8 & $3,43 \%$ \\
\hline Univ Autonoma Madrid & 8 & $3,43 \%$ \\
\hline Univ Sao Paulo & 7 & $3,00 \%$ \\
\hline Univ Puerto Rico & 7 & $3,00 \%$ \\
\hline Univ Complutense Madrid & 7 & $3,00 \%$ \\
\hline Univ Sheffield & 6 & $2,58 \%$ \\
\hline Univ Brasilia & 6 & $2,58 \%$ \\
\hline Univ Politecn Catalunya & 5 & $2,15 \%$ \\
\hline Univ Politecn Madrid & 5 & $2,15 \%$ \\
\hline Univ Estadual Campinas & 5 & $2,15 \%$ \\
\hline Univ Fed Rio Grande Sul & 5 & $2,15 \%$ \\
\hline Smithsonian Trop Res Inst & 5 & $2,15 \%$ \\
\hline
\end{tabular}
el apartado delas instituciones. El autor más productivo Ilegaalos 10 trabajos, y en su caso sedaun hecho curioso: 
posee más trabajos que su propiainstitución (7) debido a quefirma en tres ocasiones con otra: $\mathrm{U}$ niv $\mathrm{N}$ ova L isboa. Este es el caso de los cambios de instituciones de $M$ acías $C$ hapula. A continuación van apareciendo escalonadamente autores de diversas instituciones, aunquese notaun predominio deC SIC/CIN DOC en particular y deEspaña en general.

\section{CITASPOR AUTOR}

En la figura 8 (apéndice) y el cuadro 6 encontramos los autores más citados, siendo la gran mayoría de ellos anglosajones. En el cuadro hemos detallado los iberoamericanos (en cursiva y subrayados). C omo se puede observar no existe una correlación directa entre el nivel deproducción y el decitación, en gran medida porque no hemos tenido en cuenta las autocitas. A I igual que en los puntos anteriores, en la citación de autores se aprecia una clara tendencia hacia aquellos que proviene del mundo de la bibliometría. Se destaca el nivel de citación del chileno K rauskopf, el primero de los dela región.

\section{CITASPOR REVISTAS}

En el caso de la citación por revistas, podemos observar una amplitud temáticamayor queen el caso delacitación por autores. El campo bibliométrico sigue siendo preponderantecon su publicación insignia, Scientometrics, queacumula más del $15 \%$ delas citas. Sin embargo, detrás de ella aparece el JA SI S como segunda revista citada, aunqueno sea lasegundaen producción.

D el cuadro 7 debemos destacar dos grupos de revistas destacadas. El primero está compuesto por aquellas publicaciones, generalmente revistas ISI, que no pertenecen a la categoría $B \& D$ pero que son frecuentemente citadas. Estas revistas indican la exportación que se hace desde otras disciplinas hacia la $B \& D$. Tal es el caso de las revistas: $L$ ancet, $A r c h B$ iol $M$ ed Exp, J ama y B rit M ed J (M edicina), J Pers Soc Psychol y B ehavioral $\mathrm{B}$ rain $\mathrm{SCI}$ (Psicología), Science, $\mathrm{N}$ ature y Recherche(M ultidisciplinarias) etc.

El segundo grupo destacable es el compuesto por la revistas iberoamericanas. L a más citada de las revistas de la región es Interciencia, lo cual no es desorprender yaque es revista fuente. Si no centramos en las restantes, nos encontramos que la publicación con mayor visibilidad internacional es laR ev Esp D ocC ient. Lagran cantidad de citas deesta revista se puede explicar por el volumen dela producción española. Bastante más atrás aparecen
CUADRO 5

\section{A utores más productivos}

\begin{tabular}{l|l|c|}
\multicolumn{1}{c|}{ Autor } & \multicolumn{1}{c|}{ Institución } & $\#$ \\
\hline Correia AMR & Inst Nacl Engn \& Tecnol Ind (Portugal) & 10 \\
\hline Gomez I & Con Sup Inves Cientif, CINDOC & 10 \\
\hline Mendez A & Univ Illes Balears & 9 \\
\hline Maciaschapula CA & Univ Colima & 8 \\
\hline Krauskopf M & Univ Austral Chile, Inst Bioquímica & 7 \\
\hline Russell JM & Univ Nacl Aut México, CUIB & 7 \\
\hline Bordons M & Con Sup Inves Cientif, CINDOC & 7 \\
\hline Campanario JM & Univ Alcalá de Henares, Dept Fisica & 6 \\
\hline Pulgarin A & Univ Extremadura, Fac Biblioteconomía & 6 \\
\hline Alvarez P & Univ Extremadura, Fac Administración & 6 \\
\hline Curras E & Univ Aut Madrid, Dept Documentación & 6 \\
\hline Fernandez MT & Con Sup Inves Cientif, CINDOC & 6 \\
Plaza LM & Con Sup Inves Cientif, CINDOC & 5
\end{tabular}

\section{CUADRO 6}

\section{A utores más citados}

\begin{tabular}{l|lll|}
\multicolumn{1}{c|}{ Autor } & \# & Callon M & 16 \\
\hline Garfield E & 73 & Arunachalam S & 15 \\
\hline Braun T & 28 & Salton G & 14 \\
\hline Krauskopf M & 26 & Small H & 14 \\
\hline Schubert A & 26 & Gaillard J & 14 \\
\hline Price DD & 24 & Brookes BC & 13 \\
Frame JD & 19 & Arvanitis R & 13 \\
\hline Narin F & 19 & Merton RK & 12 \\
\hline Latour B & 19 & Line MB & 12 \\
Lancaster FW & 18 & Belkin NJ & 11 \\
\hline Wright BD & 17 & Vinkler P & 11 \\
\hline Gomez I & 17 & Cronin B & 11 \\
\hline Narvcezberthele.N & 16 & Luukkonen T & 10 \\
\hline Moed HF & 16 & Vessuri HMC & 10
\end{tabular}

dos revistas brasileñas: Rev Biblio Brasilia y Ciência I nformação, cerrando la lista dos publicaciones españolas: B A nabad y PoliticaC ientifica. Lo queresulta extraño es que no aparezca ninguna publicación del tercer país en producción, M éxico, y cuyarevista más prestigiosa(I nvestigacion B ibliotecologica) reúneapenas dos citas. 


\section{ANÁLISISDE COCITACIÓN DE REVISTAS}

Por último hemos realizado un análisis de cocitación de revistas (ACR), con aquellas publicaciones más citadas que aparecen el cuadro 7. C on la información sobre cocitación construimos una matriz multivariante a la que le aplicamos la técnicas de escalamiento multidimensional (MDS), para obtener un mapa bidimensional queagrupe las revistas en función decómo son vistas y citadas por los autores iberoamericanos. Este mapa se puede observar en la figura 8 (apéndice).

En el hemos delimitado tres diferentes agrupaciones de revistas. La primera estácompuesta por las publicaciones relacionadas con la bibliometría y que aquí designamos demaneramás genéricacon las siglas C IB (cienciometría, informetría y bibliometría). Si bien la natural eza de esta agrupación es un tanto heterogénea, es el campo más representativo delaproducción delaregión. A quí aparece la publicación más citadaScientometricsy las más citadas de la región I nterciencia y R ev Esp D oc C ient. También la mayoría de las revistas ajenas a la categoría $B \& D$, ya sean demedicina, psicología o multidisciplinares.

La segundaagrupación lahemos denominado RI yaque la mayoría desus publicaciones están orientadas al estudio delarecuperación delainformación. La mayoría de ellas son de la categoría, aunque hay una que pertenece al campo temático de la Informática: Commun $\mathrm{Acm}$. A Igunas de estas revistas suelen publicar trabajos relacionados con labibliometría (J Am Soc I nform Sci y J I nform Sci), por ello aparecen en lafronteracon C ib. Libr Trends, en cambio, está más relacionada con el mundo bibliotecario, por ello se encuentra más cercana a la terceraagrupación.

Estatercera agrupación seencuentra menos concentrada y está compuesta por publicaciones del mundo bibliotecario y de corte profesional. Todas pertenecen a la categoría $B \& D$, con la salvedad de PhysicsT oday, una revista de física que ha sido varias veces citadas con relación a la problemática de la subscripción de publicaciones periódicas en esta área. La posición del $B$ Anabad puede explicarse no tanto porque publique trabajos bibliométricos, sino más bien porquetira deella su compatriotaR ev Esp D ocC ient, con laqueguardauna fuerte tasa de cocitación. U n caso similar es el de laRev $B$ iblio $B$ rasilia, aunque estase encuentra relacionadacon el campo de laRI por lo queel trazo de lalínea divisoria debeconsiderarse arbitrario .
CUADRO 7

R evistas más citadas

\begin{tabular}{l|c|}
\multicolumn{1}{c}{ Revista } & $\#$ \\
\hline Scientometrics & 364 \\
\hline J Am Soc Inform Sci & 133 \\
\hline J Inform Sd & 67 \\
\hline J Doc & 62 \\
\hline Interciencia & 62 \\
\hline Science & 52 \\
\hline Rev Eso Doc Cient & 46 \\
\hline Soc Stud Sci & 46 \\
\hline Inform Process Manag & 44 \\
\hline Current Contents & 43 \\
\hline Libr Trends & 29 \\
\hline Res Policy & 29 \\
\hline B Med Libr Assoc & 25 \\
\hline Libr J & 20 \\
\hline Nature & 20 \\
\hline Lancet & 20 \\
\hline MIS Quart & 20 \\
\hline Commun ACM & 17 \\
\hline Brit Med J & 17 \\
\hline Serials Libr & 16 \\
\hline
\end{tabular}

\begin{tabular}{l|l|}
\hline Tappi J & 15 \\
\hline Inform Manage & 15 \\
\hline Physics Today & 14 \\
\hline ASLIB Proc & 14 \\
\hline Coll Res Libr & 14 \\
JAMA & 14 \\
\hline Arch Biol Med Exp & 13 \\
\hline J Acad Libr & 12 \\
\hline Rev Bibllo Brasilia & 12 \\
\hline J Pers Soc Psychol & 12 \\
\hline Electron Libr & 11 \\
\hline Restaurator & 11 \\
\hline Res Evaluation & 11 \\
\hline Manage Sci & 11 \\
\hline Recherche & 11 \\
\hline Clencla Informacao & 10 \\
\hline Politica Cientifica & 10 \\
\hline Scientist & 10 \\
\hline Annu Rev Inform Sci & 10 \\
\hline B Anabad & 10 \\
\hline
\end{tabular}

Las consideraciones que pueden extraerse de esta representación son algo subjetivas, sobretodo porque los niveles de citación (y por ende de cocitación) son bastante bajos. A pesar dequecontamos con pocos datos, en líneas generales la representación guarda una coherencia interna bastante considerable y sus agrupaciones son razonables.

\section{CONCLUSIONES}

El análisis delos resultados expuestos nos permiteextraer las siguientes conclusiones sobre la visibilidad internacional de la investigación iberoamericana en el campo delaB\&D:

- A unque la distribución por países de la producción científica iberoamericana en este campo es similar a la del conjunto de las ciencias sociales, resultafácilmente constatable que algunos países han colocado en el período objeto de estudio un número detrabajos que no secorrespondecon su potencial en disciplinas similares.

- Las diferencias tan notables existentes en materia de política científica y desarrollo curricular contribuyen de forma definitiva a que la distribución de trabajos por país en el perío do 1991-2000 favorezca a los países que disponen de una mayor infraestructura académica e investigadora. Por otro lado, con la excepción del caso español, las cifras tan bajas de producción en estecampo 


\section{Félix de M oya-A negón / V íctor H errero-Solana}

científico están relacionadas con la escasa atención que recibe la investigación bibliométrica por parte de las universidades y centros de investigación latinoamericanos. Siendo estesubcampo uno delos dos más activos internacional mente.

- D esde una perspectivaglobal podemos considerar que I beroamérica ocupa una posición muy periférica en el panorama delainvestigación mundial en nuestro campo. Baste comprobar que países como $\mathrm{H}$ olanda o $\mathrm{F}$ rancia superan al conjunto dela región en cifras de producción en ese mismo periodo.

- Ladistribución institucional delainvestigación pone de manifiesto que existen dos instituciones muy productivas (UN A M y C SIC) que tienen centros de investigación. A estas les siguen un grupo de universidades, entre las que predominan las españolas, con cifras de producción sensiblemente inferiores. Este hecho tiene una gran influencia en la concentración temática delainvestigación, lo que resulta corroborado cuando observamos laproducción por revista.

- U na delas conclusiones más desalentadoras seobtiene del análisis de la producción por año en la región. La ligerísimatendencia ascendente ponede manifiesto que a pesar de nuestro bajo punto de partida, durante estos nueve años no hemos crecido como debiéramos. Probablemente sigue habiendo una clara inclinación localista por parte de los investigadores en $B \& D$ que prefieren difundir los resultados desus trabajos através delas publicaciones dela región.

- El análisis delas fuentes utilizadas, tanto desdeel punto de vista de los autores como desde las revistas citadas, confirmael sesgo temático en favor delabibliometría de la investigación $B \& D$ difundida internacionalmente, lo que favorece la presencia internacional de aquellas instituciones que cuentan con investigadores en esa especialidad.

- U n estudio máspreciso delacitación ponedemanifiesto que existen tres grandes áreas de actividad en este conjunto de trabajos: bibliometría(CIB), bibliotecas y recuperación deinformación ( $\mathrm{RI})$. A unque existe una cierta similitud entre esta caracterización temática y la de la investigación ISI, es en el área de CIB en la que podemos encontrar mayor proporción detrabajos.

A rtigo aceito para publicação em 19-05-2002

\section{REFERÊNCIAS}

1. WHITE, H oward D.; MCCAIN, Katherine W. Visualizing a discipline: an author co-citation analysis of information science, 1972-1995. J ournal of the A merican Society for I nformation Science, v. 49, n. 4, p. 327-355, 1998.

2. ; LIN , Xia; McCAIN , Katherine W. Two modes of automated domain analysis: multidimensional scaling vs. kohonen feature mapping of information science authors. Structures and relations in knowledge organization. I n: I N TER N ATI O N AL ISK O C O N FEREN C E, 5th, 1998. Proccedings... [S. I. : s. n.], 1998.

3. MOYA-ANEGÓ N, Félix; JIMÉNEZ CONTRERAS, Evaristo; HERRERO SO LAN A, Víctor. 1999. A connectionist approach to science maps: SO M and clustering techniques applied to Library \& Information Science research, 1992-1997. In: INTERNATIONAL CONFEREN CEON SCIENTOMETRICS AN D INFORMETRICSINTERNATIONAL SO CIETY FOR SCIEN TO METRIC SAN D IN FO RMETRIC S, 7 th, 1999.

4. PERSSO N, O lle. The intellectual base and research fronts of JA SI S: 1986-1990. J ournal of the American Society for I nformation Science, v. 45, p. 31-38, 1994.

5. JIMÉNEZ CONTRERAS, Evaristo; MOYA-A N EG Ó N, Félix. A nálisis de la autoría en revistas españolas de biblioteconomía y documentación, 1975-1995. R evista E spañola de D ocumentación C ientífica, v. 20, n. 3, p. 252-266, 1997.

6. MOYA-ANEGÓN, Félix; JIMÉNEZ CONTRERAS Evaristo; MONEDA CORRO CHANO, Mercedes de la. Research fronts in library and information science in Spain (1985-1994). Scientometrics, v. 42, n. 2, p. 229-246, 1998.

7. Félix; H ERRERO SO LAN A, Víctor. 1999a. A nálisis de dominio de la investigación bibliotecológica mexicana. I nformación C ultura y Sociedad. n. 6, 2001.

8. GÓ MEZ, I sabel et al. Influence of Latin A merican journals coverage by international databases. Scientometrics, v. 46, n. 3, p. 443-456, 1999.

9. MOYA-AN EG Ó N, Félix; H ERRERO SO LAN A, Víctor. Science in A merica Latina: a comparison of bibliometric and scientifictechnical indicators. Scientometrics, v. 46, n. 2, p. 299-320, 199b 
V isibilidad internacional de la producción científica iberoamericana en biblioteconomía y documentación (1991-2000)

\section{A péndice}

\section{Lista de revistas fuente}

\begin{tabular}{|c|c|c|c|c|}
\hline Abroviatura & Titulo & ISSN & Pais & Idioma \\
\hline AMARCHIVIST & Ametiean Atehivist & $0360-9081$ & USA & Ing \\
\hline ANNU REV INFORM SCI TECH & Annual Review of Irformation Science and Technology & 0060.4200 & USA & Ing \\
\hline ASLIBPROC & Aslib Proceedtings & $0001.253 \mathrm{X}$ & UK & Ing \\
\hline BMED LIBR ASSOC & Bulletin of The Medical Libray Association & 0025.7338 & USA & Ing \\
\hline BEHAV SOC SCI LIER & Behawioral \& Social Sciences Librarian & $0163-9269$ & USA & lng \\
\hline CAN IINPCRMI SCI & Candian Joumal of Information Science & \multicolumn{3}{|c|}{ Cont: Can Inferm Lib Sci } \\
\hline CAN IINFORM LIB SCI & Conatian Joumal of Intornation And Library Science & $1105-006 \mathrm{x}$ & Conads & Ing-Fra \\
\hline CD ROM PFOF & Cd-Ron Protessional & $104 \% \cdot 0833$ & USA & Ing \\
\hline COLL RES LIBR & College \& Research Libraties & 0010.0870 & USA & Ine \\
\hline DATABASE & Database & \multicolumn{3}{|c|}{ Cont Econtent } \\
\hline BCONTENT & Econtent & 0102.4105 & USA & Ing \\
\hline EDUCINFCRM & Edusation for: Intormation & 0167.8329 & Holanda & Ine \\
\hline ELECTRON LIER & Electronie Libraty & 0264.0473 & UK & Ine \\
\hline OOVTINFORM QUART & Covennent Information Quaterly & $0740.624 \mathrm{X}$ & USA & Ing \\
\hline CONT PUBL FEV & Govemment Publisations Review & \multicolumn{3}{|c|}{ Cont: J Govement Inionmation. } \\
\hline IFLA J INT FED LIBR ASS & IF_.A Journa] & 03400352 & Alemania & Ale-Fun-Ing \\
\hline INFORM MANACE & Infotmation Management. & 0019.9966 & Hdanda & Ine \\
\hline INFORM FROCESS MANAG & Infotmation Procesting \& Management & 03064573 & USA & Ing \\
\hline INFORM SYST RES & Infotmation Systems Research & 1047.7047 & USA & Ing \\
\hline INFORM TECHNOL LIBR & Information Techndogy and Libranes & 07309295 & USA & Ine \\
\hline INT CLASS & Intemational Classification & \multicolumn{3}{|c|}{ Cont: Knowl Organ } \\
\hline INT PORUM INFORM DOC & Intenaticnal Forum an Information and Documentation & $0304-9701$ & Holande & Ing \\
\hline INT INF LIBR REV & The International Information and Liftaty Review & $1057 \cdot 2317$ & UK & Ine \\
\hline INT J CEOCR INE SYST & Intemational Journal of Ciecgrophical Infoumation Sistems & 0269.3798 & UR & Ing \\
\hline INT I INFORM MANACE & Intertiational Journal of Information Marngenent & 0268.4012 & UK & Ing \\
\hline INT LIBE REV & Intelnational Librany Review & \multicolumn{3}{|c|}{ Cont: Int Inflibr Rey } \\
\hline INTERCIENCIA & Inretciencia & $037 \& 1844$ & Venesuela: & Esp-Pordine \\
\hline INTERL FND DOC SUFFIY & Intertending and Document Surpply & 0264.1015 & UK & Ing \\
\hline I ACAD LIER & The Joumal of Acaderni- Libratianship & 0000.1333 & USA & Ins \\
\hline JDOC & Joutral of Documsentation & $0022-0418$ & UK & Ing \\
\hline 1EDUC LIBR INF ORM SCI & Toumal of Eduration for Libraty and Information Science & 07485786 & USA & Ing \\
\hline JINECRAS SCI & Fouthal of Informatisn Seitnce & 1352.7450 & UK & Ing \\
\hline JLIER INF SCT & Joutnal of Library and Information Science & $0970-714 \mathrm{x}$ & Indis & Ing \\
\hline IOOVERNMENT INFORMATION & Toumal of Govemment Intormation & $1352-0232$ & USA & Ine \\
\hline J AMER MFD INFCRM ASSOC & Joumal of the American Mectical Informatics Association & 1067.5027 & USA & Ing \\
\hline J AB SOC INFORM SCT & Joutnal of The American Society for Information Science & $0002-8231$ & USA & Ing \\
\hline J SCHOLARLY PUBLISHING & Joumal of Scholady Publishing & 11989742 & Canacka & Ing \\
\hline KNOWL ACQUIS & Kuowledec Acquisition & $1042-8143$ & UK & Ine \\
\hline KNOWL OKGAN & Knowle-dge Organization & .0943 .7444 & Alemania & Ale-Fra-Ing \\
\hline LAW LIBR J & Law Libraty Jouttial & 00239283 & USA & Ing \\
\hline LIER ACQ FKACT THEO & Library Accuisitions: Fractice and Theory & \multicolumn{3}{|c|}{ Cont Libr Collect Acquns Tech Setv } \\
\hline LIAR COLLIECT ACQUIS TECH SERV & Library Collections, Acquisitions, and Technical Services & 1404.9055 & UK & Ing \\
\hline LJER CULTURE & Libraries \& Culture & $0894-8631$ & USA & Ing \\
\hline LIER HI TECH & Lubravy Hi Tech & $0737-8831$ & USA & Ing \\
\hline LIBR INFORM SCA & Library and Information Science & 03734447 & Japón & Ing-Jap \\
\hline LIRR INFORM SA RES & Libraty \& Information Science Research & 0740.8138 & USA & Ing \\
\hline LIER J & Library Joutnal & $0363-0277$ & USA & Ing \\
\hline LJER QUART & Libraty Quarterly & $0024-2519$ & USA & Ing \\
\hline LIRR RESOUR TECH SER & Library Resources \& Tedinical Services & 0024.2527 & USA & Ine \\
\hline LIAR TRENDS & Libraty Tiends & 00242594 & USA & lne \\
\hline LIERI & Libri & 0024.2007 & Dinamarea & Ale-Fra-Ing \\
\hline NACHR DCK & Nochrichten tiuet Dolsumentation & \multicolumn{3}{|c|}{ Cont: N F D Information } \\
\hline NAUCHN TEKHN INFOKM SER & Nauchno-Tekhnicheskaya Infotmatsiva Setiya & 05480019 & Fusia & Rus-Ine \\
\hline ONLINE & Coline & 01465422 & USA & lne \\
\hline CNLINE CDROM REV & Online \& Cduom Review & 1353.2642 & USA & Ing \\
\hline
\end{tabular}

Ci. Inf., Brasília, v. 31, n. 3, p. 54-65, set./dez. 2002 
FIGURA 1

Producción en B\&D en el contexto del SSC I

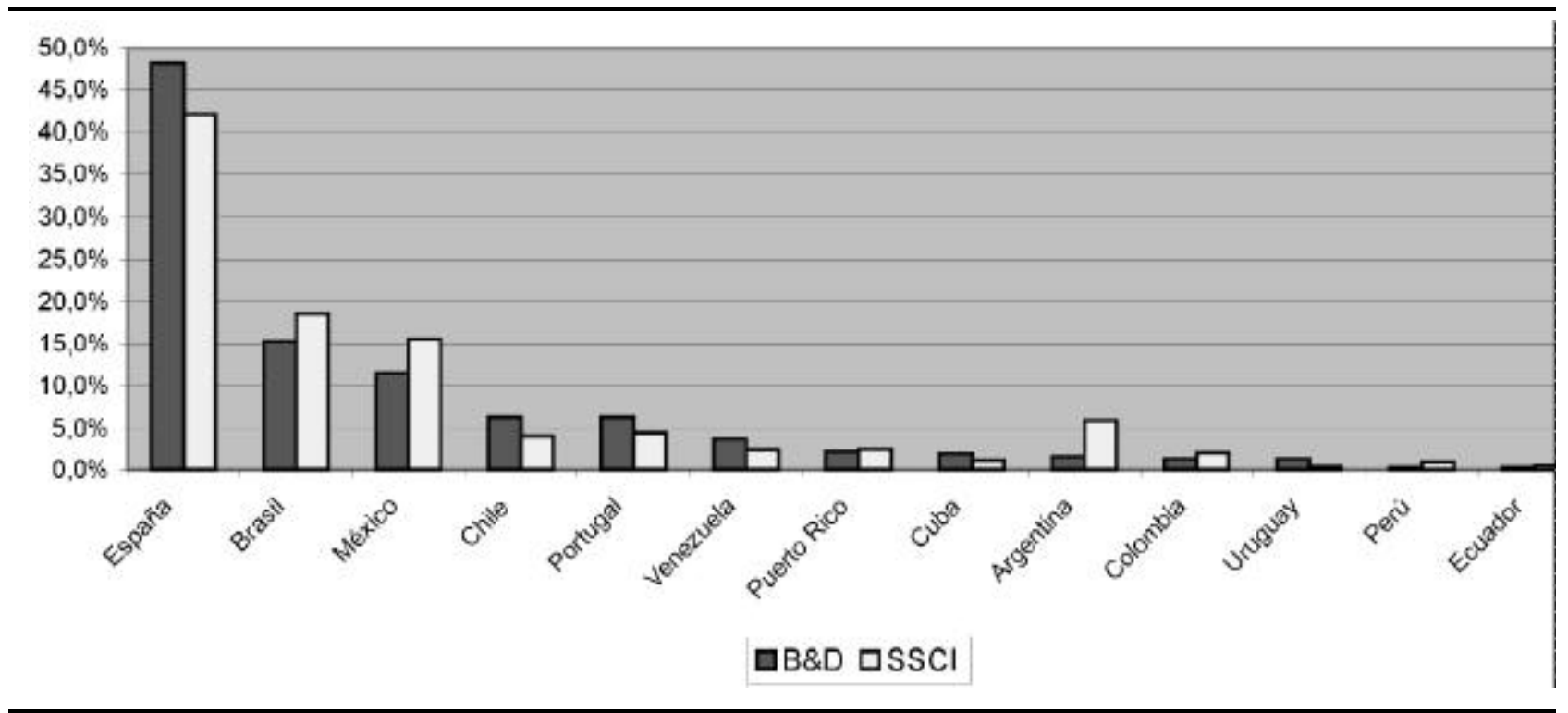

FIGURA 2

Producción por países en B\& D-SSC I

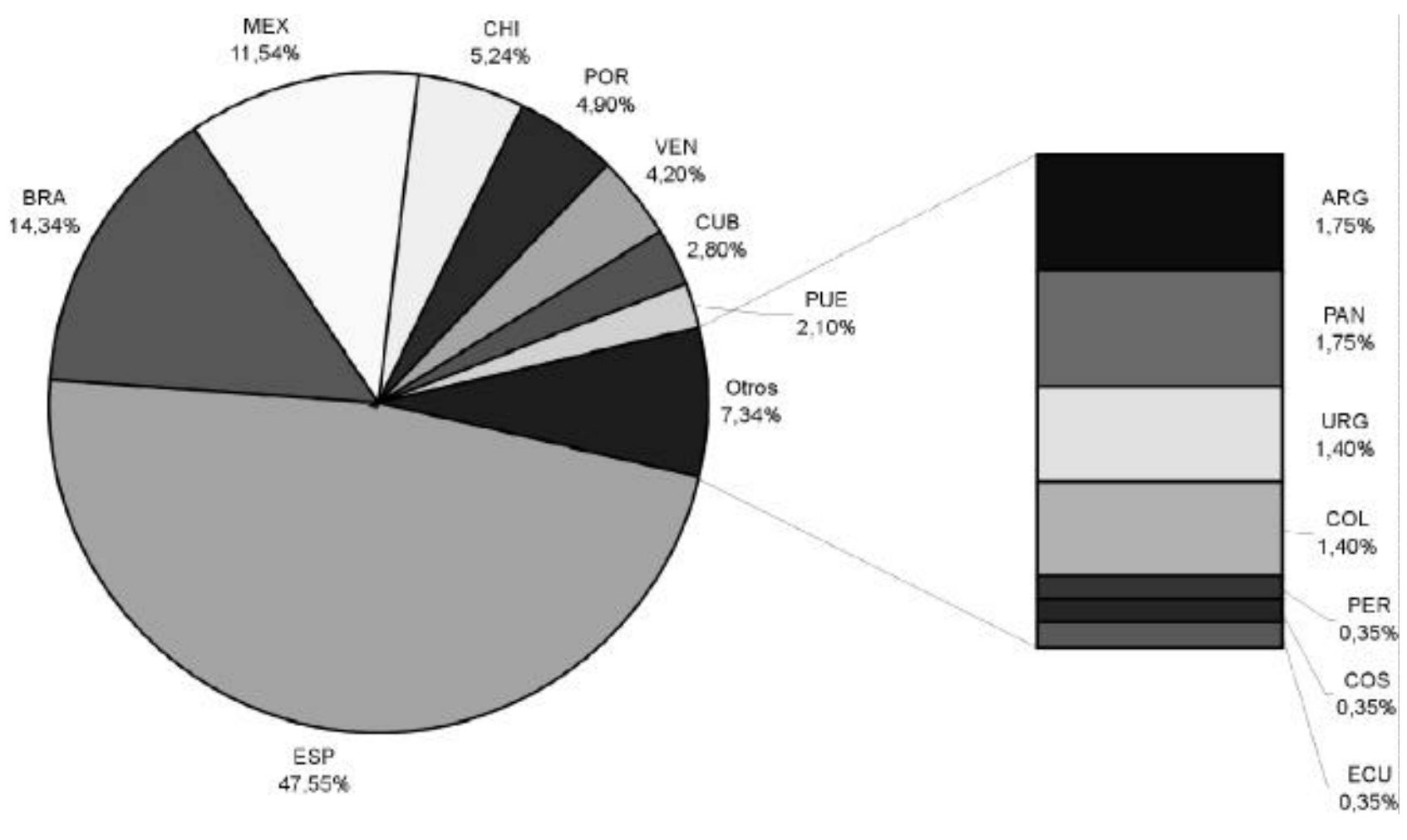




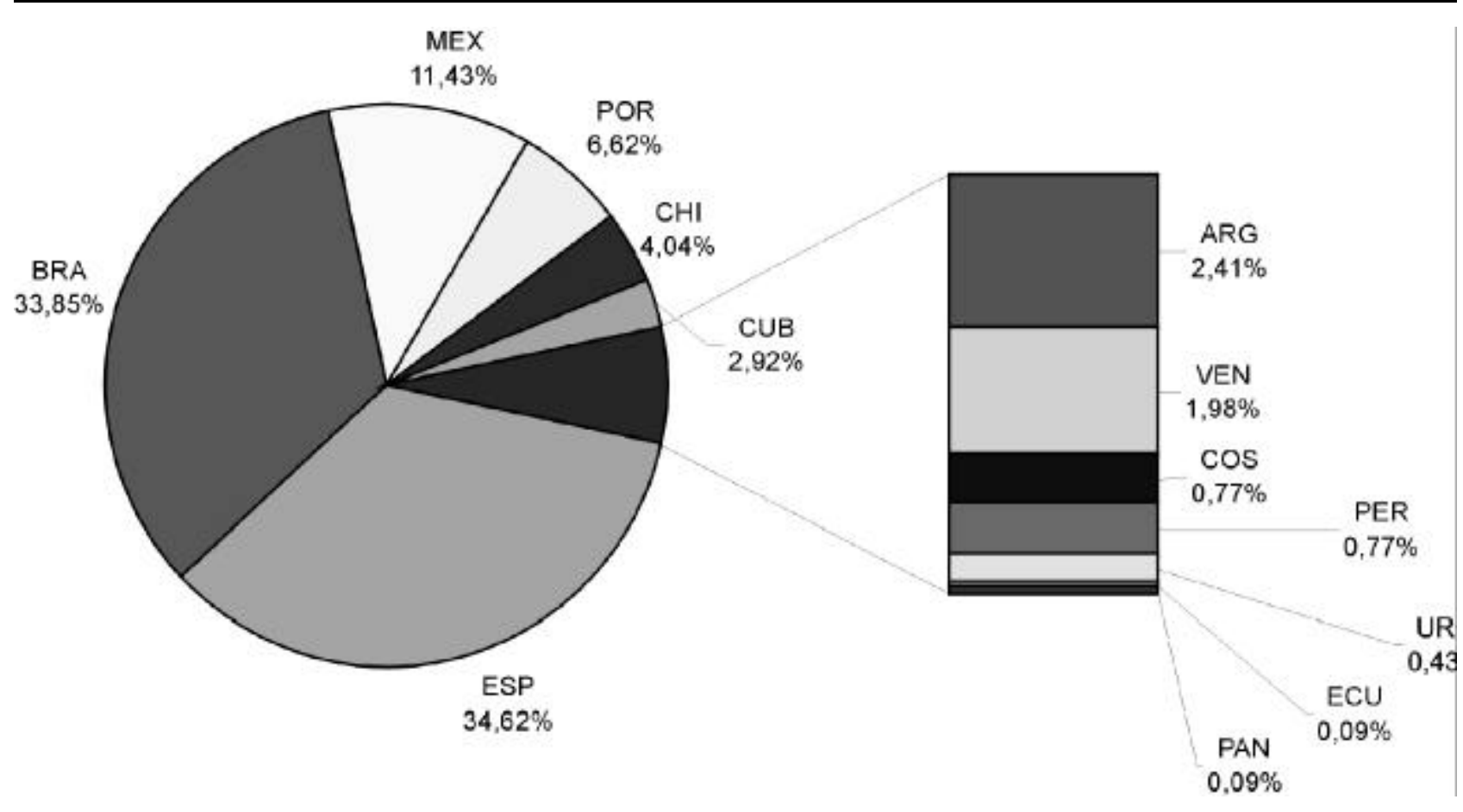

FIGURA 4

Distribución de la producción en ISA por grandes regiones

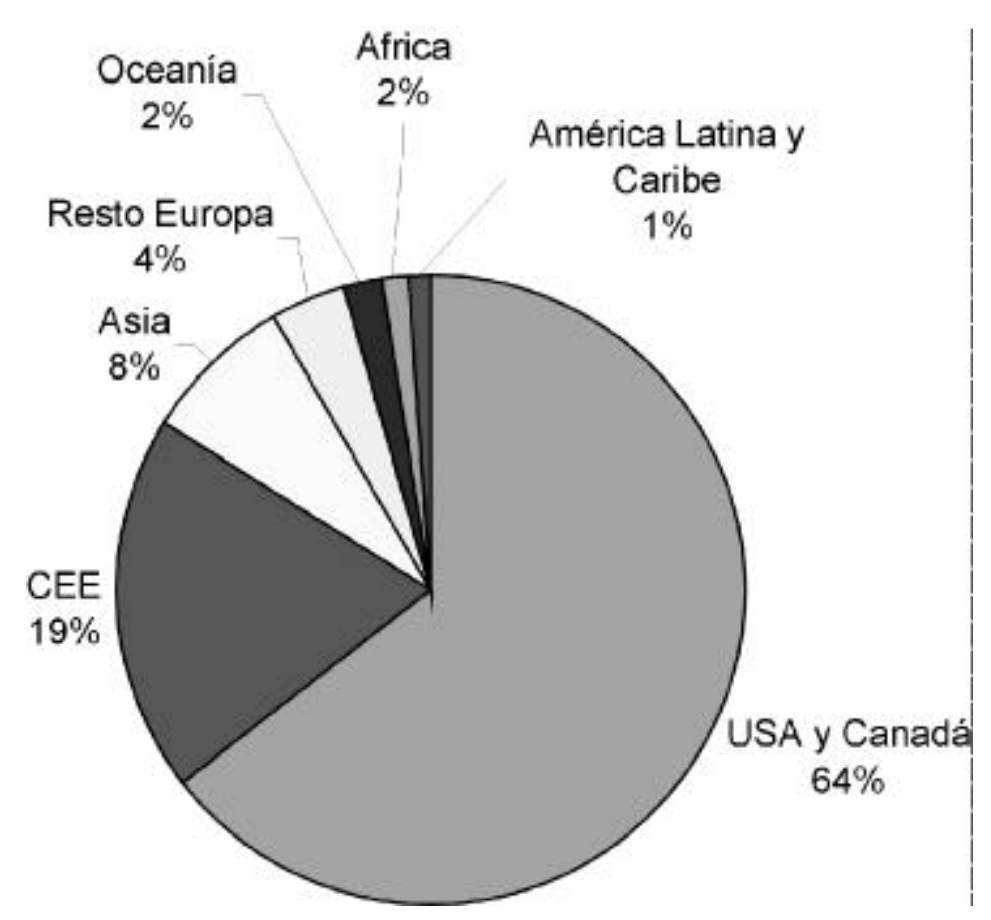


FIGURA 5

Producción por instituciones

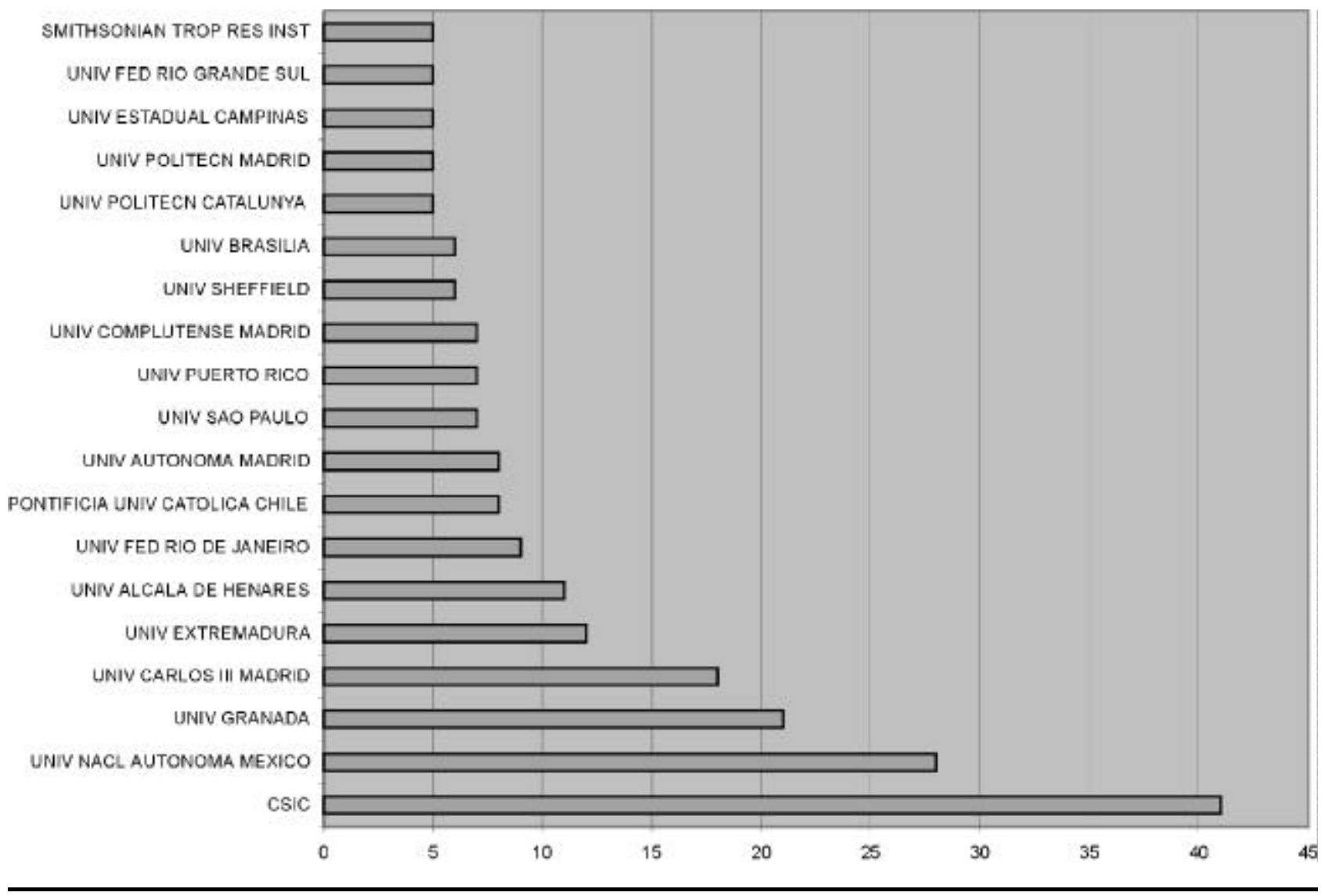

FIGURA 6

\section{Producción por revistas}

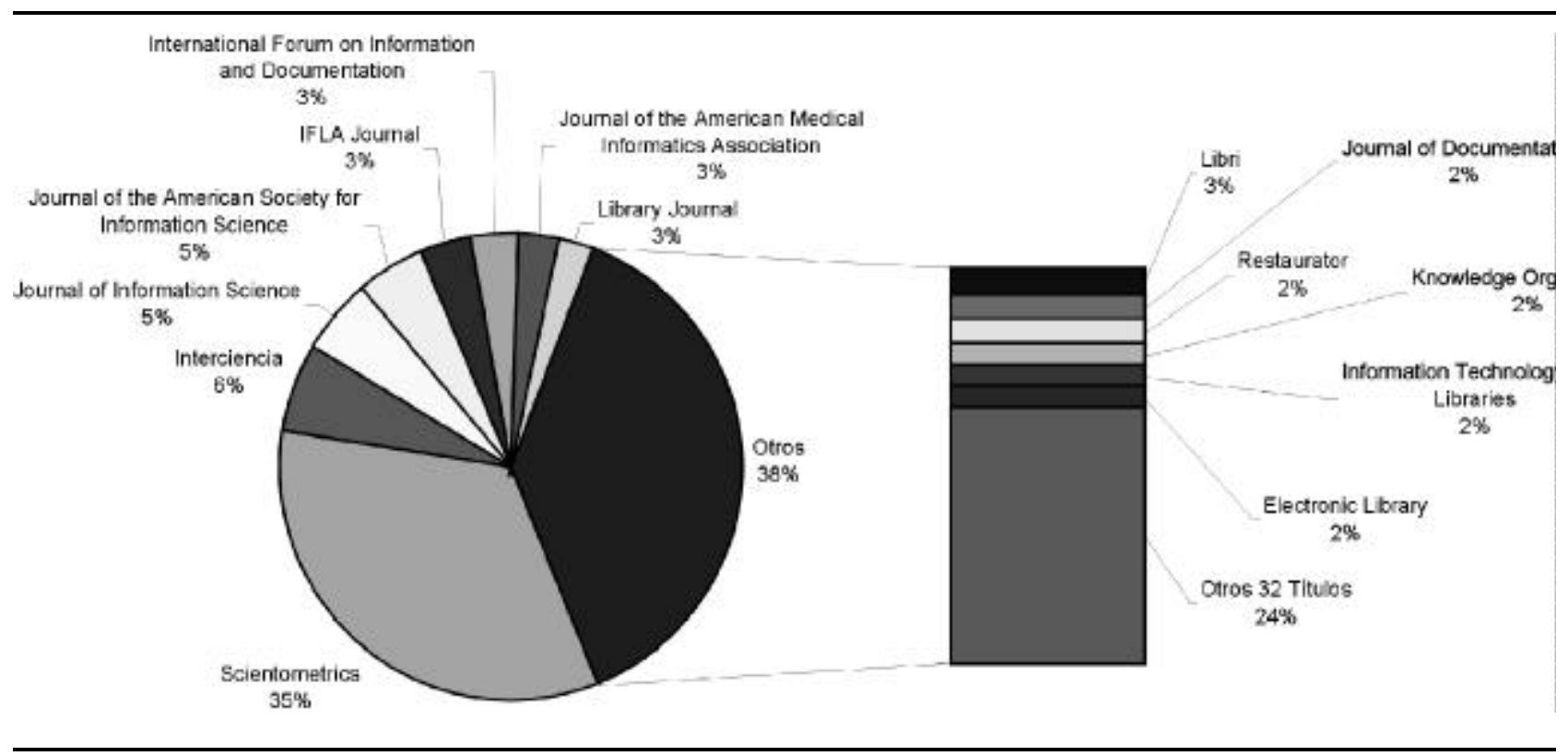


Evolución histórica de la producción regional

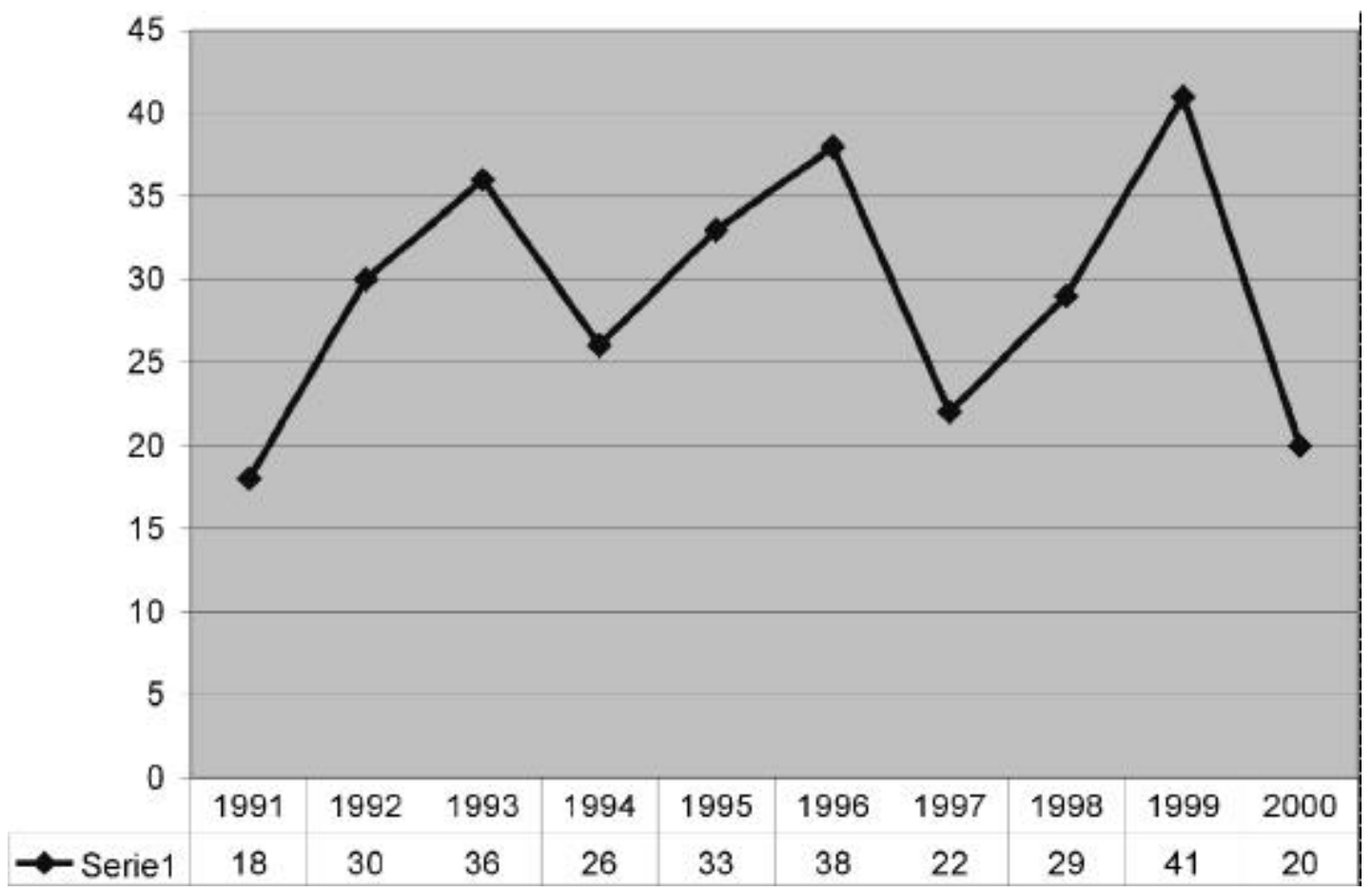

FIGURA 8

Mapa de cocitación de revistas (AC R )

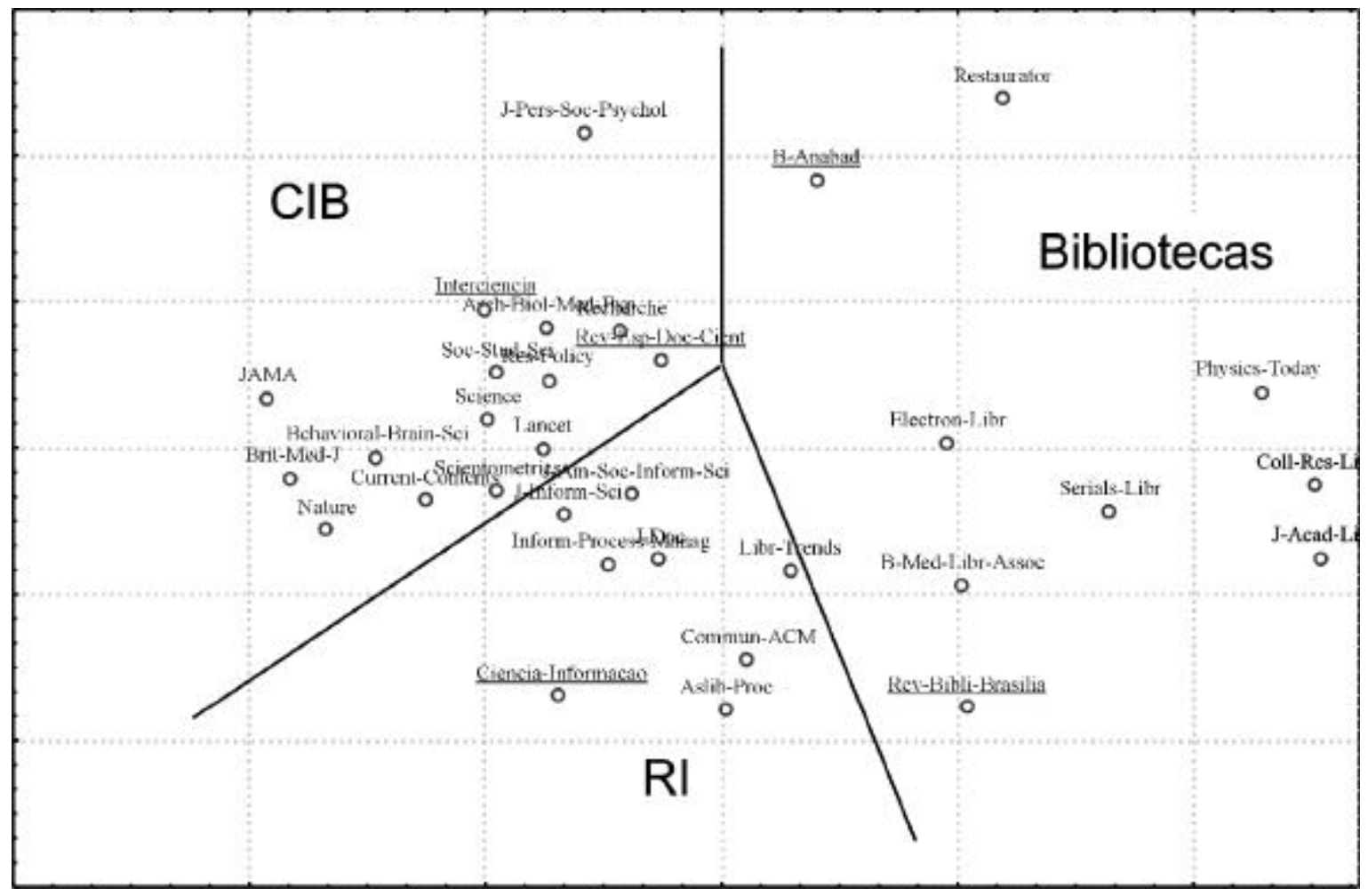

Ci. Inf., Brasília, v. 31, n. 3, p. 54-65, set./dez. 2002 